\title{
RECUENTO DE OOQUISTES DE EIMERIA SP. EN CAMA NUEVA Y SU RELACIÓN CON LA PIGMENTACIÓN EN POLLOS DE CARNE
}

\author{
Patricia Caiña V.1, Eliana Icochea D’A. ${ }^{2}$, Pablo Reyna S.', Amanda Chávez ${ }^{3}$, Eva \\ Casas A. ${ }^{3}$ y María Elena Salinas J. ${ }^{1}$
}

\section{The relationship between Eimeria sp. oocyst contamination in litter and broiler pigmentation}

The objective of this study was to determine the relationship between avian coccidiosis and pigmentation intensity in broiler chickens kept under intensive husbandry conditions. Litter samples were taken on days $0,7,14,21,42$ and 49 at 5 farms located in Huaral, Huacho and Ventanilla, Department of Lima. On day 35, skin pigmentation was determined using a Roche color fan, for $90-120$ birds from each farm. A weak relationship between level of oocyst contmination and broiler skin pigmentation intensity was found (Spearman correlation -0.098), although subclinical coccidiosis was detected in all the poultry houses studied. Clinical signs of the disease, which may adversely affect pigmentation and productive parameters, were not observed due to appropriate management and biosecurity practices, chicken health status and the anticoccidial program used.

Key words: Coccidiosis, broiler, pigmentation.

El presente estudio tuvo por objetivo determinar la relación entre la coccidiosis aviar e intensidad de pigmentación en los pollos de carne criados en forma intensiva. Se tomaron muestras de cama los días $0,7,14,21,35,42$ y 49 de 5 granjas ubicadas en las zonas de Huaral, Huacho y Ventanilla. Se utilizaron entre 90 a 120 animales por cada granja para medir el grado de pigmentación de las aves el día 35 de cada campaña, utilizando para ello el abanico colorimétrico de Roche. La relación entre el grado de contaminación de cama e intensidad de pigmentación de la piel, fue analizada mediante la prueba de correlación de spearman, obteniéndose una correlación débil de -0.098 . El presente estudio nos permite concluir que la relación entre el grado de contaminación de ooquistes en cama e intensidad de pigmentación de la piel del ave, fue baja. Adicionalmente aún cuando en todos los galpones evaluados se encontró presencia de coccidiosis subclínica, el correcto manejo, programa anticoccidial, bioseguridad y el estado de salud de las aves no permitieron la presentación de manifestaciones clínicas de la enfermedad, que afecten la pigmentación y los parámetros productivos.

Palabras clave: Coccidiosis, pollos y pigmentación.

\footnotetext{
'Práctica privada.

${ }^{2}$ Laboratorio de Patologia Aviar- FMV-UNMSM

${ }^{3}$ Laboratorio de Parasitología - FMV-UNMSM
} 


\section{6.}

En el Perú, así como en algunos países de Latinoamérica y del mediterráneo, se da importancia a la intensidad del color de la piel del ave, debido a que es comúnmente asociada con un animal saludable. En animales que se alimentan con maíz amarillo o que tienen dietas suplementadas con agentes pigmentantes, la pobre pigmentación de la piel está relacionada a una disminución en la absorción de carotenoides a nivel del duodeno y yeyuno, lo que puede deberse tanto a problemas tóxicos alimenticios como a la coccidiosis aviar.

En pollos de carne, la prevención de la coccidiosis está orientada al uso de tratamientos profilácticos, para lo cual se adiciona coccidiostatos al alimento impidiendo así la presentación de coccidiosis clínica y subclínica, sin embargo, constantemente se presentan en el campo cuadros de coccidiasis que si bien no causan mortalidad afectan los parámetros productivos y la pigmentación de la piel. Debido a los problemas de resistencia que enfrenta el uso continuo de anticcocidiales, las empresas avícolas emplean programas de rotación de drogas, las cuales son evaluadas continuamente mediante diversos métodos, siendo el recuento de ooquistes en cama uno de los más usados en estos casos, el cual nos da una idea sobre el estado de exposición en que se encuentra la granja y la eficacia con que está actuando el programa anticoccidial empleado.

En nuestro país, al igual que en el resto del mundo, la coccidiosis produce fuertes pérdidas económicas, no sólo por que influye negativamente sobre los parámetros productivos, sino también, por la cantidad de dinero que se invierte en anticoccidiales y agentes pigmentantes.

El objetivo del presente trabajo fue determinar la relación que existe entre la concentración de ooquistes de Eimeria sp. presentes en la cama, y el grado de pigmentación de pollos de carne.

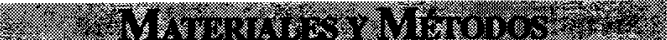

\section{Lugar de estudio}

El estudio se realizó en cinco granjas o planteles localizadas en las zonas de Huaral (B, C y D), Huacho (A) y Ventanilla (E). Las muestras fueron tomadas desde fines de marzo hasta mediados de junio.

\section{Tamaño muestral}

Siendo por primera vez realizado este tipo de trabajo en el país, el tamaño muestral se estimó en base al estudio realizado en la Universidad de Georgia por Reyna $e t$ al.(1983), donde tomaron 3 galpones por granja, es decir se trabajo con 15 galpones en 5 granjas.

\section{Camas}

Para el presente trabajo se tomaron muestras de cama de los días $0,7,14,21,28$, 35 y 42 de las granjas ubicadas en las zonas de Huaral, Huacho y Ventanilla.

\section{Animales}

Se utilizaron un promedio de 120 animales por granja, para medir la pigmentación del ave el día 35 de cada campaña. Las aves fueron alimentadas con el mismo tipo de alimento, procedente de los molinos de la empresa.

\section{Equipos de laboratorio}

Los equipos de laboratorio que fueron utilizados, son los siguientes: Microscopio, pipetas del tipo pasteur, cámara Mc Master, envases, balanza, mortero y tamiz.

\section{Reactivos}

Los reactivos fueron: $\mathrm{NaOH}$, solución sobresaturada de CINa. 


\section{Obtención de las muestras de cama}

Las muestras de cama fueron tomadas usando el método de la W invertida; el cual consiste en trazar $2 \mathrm{~W}$ opuestas en el área del galpón. Después se cuentan los pasos de c/u de las $\mathrm{W}$ y se dividen los mismos entre 50. El resultado indica el número de pasos que se debe recorrer para tomar la muestra en cada caso. Luego se toma un puñado de la cama de los cuatro costados y se deposita en una bolsa, mezclándose finalmente las camas colectadas en las $2 \mathrm{~W}$ en una cantidad que va entre 1 a $3 \mathrm{Kg}$ las cuales fueron llevadas a analizar (Rojas, 1990).

\section{Procesamiento de las muestras}

Las muestras de cama fueron analizadas por el método McMaster modificado (Rojas, 1990). Este método consiste en mezclar 100 gramos de la cama con una solución de $\mathrm{NaOH}$ al 4\% (1 litro), removiendo bien la mezcla y dejando que ésta repose hasta el día siguiente. Luego se filtra la mezcla y de este filtrado se toma $30 \mathrm{ml}$, el cual es completado con $15 \mathrm{ml}$ de agua corriente; prosiguiendo luego a dejar sedimentar por 30 minutos. Concluido el tiempo se elimina el sobrenadante y se agrega al sedimento CINa (42 ml aprox.), luego se mezcla y se coloca una pequeña cantidad en la cámara McMaster para la lectura.

\section{Recuento de Ooquistes}

La lectura de las muestras en el microscopio se realizó con un aumento de $100 \mathrm{x}$. La cantidad de oocistos encontrados en la cámara McMaster fue multiplicado por 100 , debido a que se realizó la lectura en una sola cámara.

\section{Medición de la intensidad de la pigmen- tación}

Para la evaluación de la pigmentación se utilizó el abanico colorimétrico de Roche en el día 35 de la campaña, y en una cantidad que fluctuó entre 90 a 120 animales por plantel. Esta evaluación consistió en tomar a cada animal y comparar el color de las patas del ave con las diferentes intensidades de color del abanico colorimétrico, las que fluctuaron entre un amarillo suave a un anaranjado intenso (1-15).

\section{Parámetros}

Para un mejor entendimiento del estudio se tomaron datos adicionales como el índice de conversión, peso y mortalidad por cada galpón estudiado, además de temperatura y humedad de cada plantel.

\section{Análisis de datos}

Se utilizó la prueba de Correlación de Spearman, para determinar el grado de asociación existente entre la contaminación de la cama nueva (variable independiente), y la intensidad de pigmentación de la piel del pollo (variable dependiente).

Se utilizó la prueba de Kolmogorov Smirnov para determinar la relación entre el sexo y la pigmentación de la piel del pollo de carne.

Se utilizó la prueba de Kruskall Wallis para determinar diferencias entre la pigmentación de los planteles estudiados.

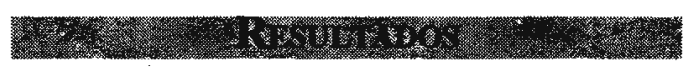

En el Cuadro 1, se puede observar que 4 de los 5 planteles en estudio, presentaron en el día cero muy baja contaminación de ooquistes, siendo el plantel $\mathrm{A}$ el único que manifestó una moderada cantidad de ooquistes al inicio de la toma de muestra. Posteriormente la concentración de ooquistes en cama fue aumentando hasta alcanzar su máximo nivel entre la 4ta. y 6 ta. semana de la campaña.

Se observó además que los planteles que presentaron más tempranamente su pico de contaminación (C, D y E), obtuvieron aparentemente animales con menor pigmentación comparándolos con aquellos que tuvie- 
Cuadro 1. Recuento de ooquistes por gramo de cama de los planteles de pollos de carne evaluados en la zona norte de Lima, 1999.

\begin{tabular}{ccccccccc}
\hline $\mathrm{N}^{\circ}$ de & \multicolumn{7}{c}{$\mathrm{N}^{\circ}$ promedio de ooquistes/g cama por semana } \\
\cline { 2 - 9 } plantel & 0 & 1 & 2 & 3 & 4 & 5 & 6 & 7 \\
\hline & & & & & & & & \\
Plantel A & 717 & 417 & 583 & 1117 & 2067 & 2167 & 3516 & 2450 \\
Plantel B & 0 & 83 & 317 & 1016 & 1450 & 3917 & 4450 & 1500 \\
Plantel C & 50 & 100 & 567 & 1533 & 3200 & 2217 & 1217 & 750 \\
Plantel D & 67 & 217 & 550 & 1417 & 3400 & 2250 & 1683 & 783 \\
Plantel E & 50 & 33 & 200 & 583 & 1883 & 1733 & 1152 & 600 \\
\hline
\end{tabular}

ron su pico de contaminación a los 42 días (Figura 1 y Cuadro 2).

Aunque numéricamente en algunos galpones los machos mostraron mayor pigmentación que las hembras (Cuadro 2), estadísticamente no se observó influencias del sexo sobre la pigmentación (Kolmogorov Smirnov de II muestras) (Daniel, 1996).

Existe diferencia estadística significativa entre las granjas estudiadas $(\mathrm{P}<0.05)$, excepto entre los planteles $\mathrm{B}$ y $\mathrm{C}$ los cuales presentaron un grado de pigmentación semejante (Kruskall Wallis) (Daniel, 1996).
No se encontró una correlación significativa entre la pigmentación de la piel y el número de ooquistes por $\mathrm{g} / \mathrm{cama}$, esto se pudo apreciar mediante la prueba de Spearman, donde se obtuvo un resultado de $r=-0.098$.

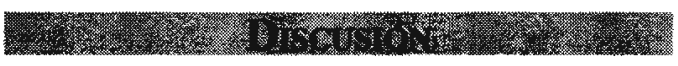

Un factor muy importante en los productos alimenticios de origen animal es el aspecto, en el caso de las aves de carne el color amarillo de la piel y la yema de los huevos son caracteres muy importantes para el intermediario y el consumidor los que re-

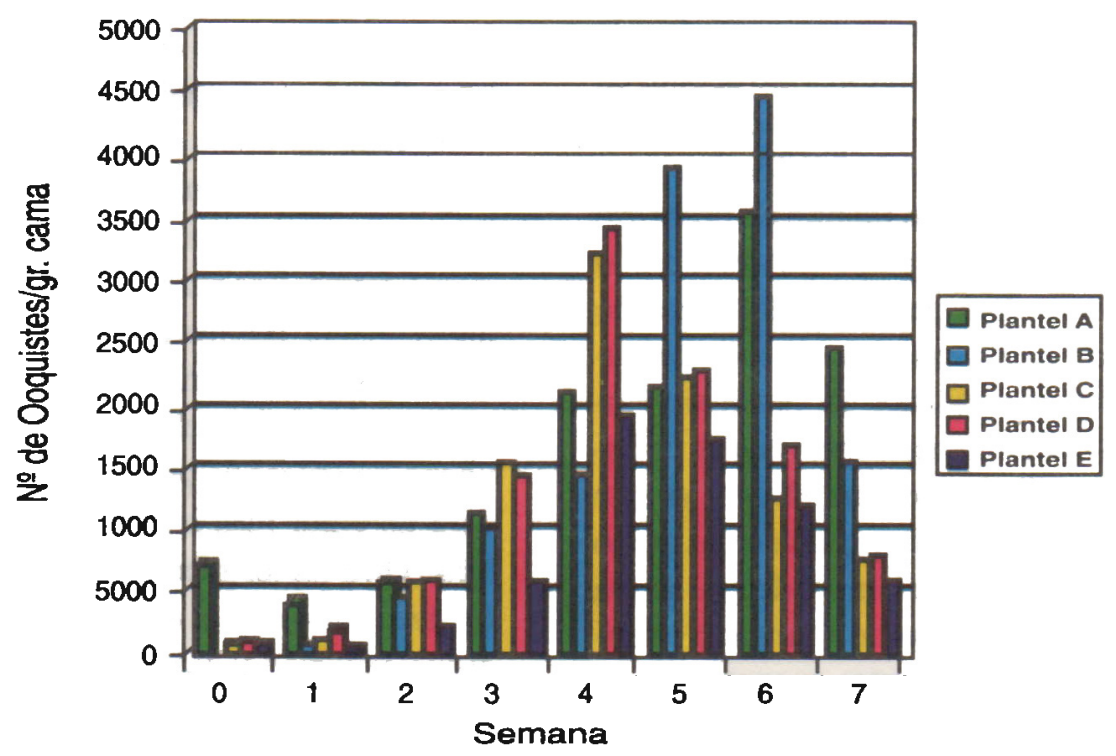

Figura 1. Recuento de Ooquistes en cama. 
Cuadro 2. Grado de pigmentación promedio en pollos de carne hembras y machos evaluados a la 5ta. Semana de edad en la zona norte de Lima, 1999.

\begin{tabular}{cccc}
\hline \multirow{2}{*}{ Plantel } & \multicolumn{2}{c}{ Grado de Pigmentación } & $\begin{array}{c}\text { Promedio de Pigmentación } \\
\text { por Plantel }\end{array}$ \\
\cline { 2 - 3 } & Hembras & Machos & \\
\hline A & 2.96 & 300 & 2.98 \\
B & 2.43 & 2.52 & 2.48 \\
C & 1.37 & 1.41 & 1.39 \\
D & 2.47 & 2.21 & 2.34 \\
E & 2.17 & 2.27 & 2.22 \\
\hline Promedio & 2.28 & 2.28 & 2.28 \\
\hline
\end{tabular}

lacionan palidez o mala pigmentación con enfermedad o inadecuada nutrición, originando el rechazo del producto.

En el presente estudio se obtuvo que cuatro de los cinco planteles al inicio de la crianza, presentaron muy baja cantidad de ooquistes por gramo de cama (Cuadro 1), a diferencia del plantel restante que si tuvo una cantidad moderada de los mismos. La concentración de ooquistes en el galpón es dependiente de condiciones de manejo y limpieza que se den en él, comúnmente se observa que aún cuando las camas son eliminadas al finalizar cada campaña, siempre queda un poco de la misma en el piso que generalmente es de tierra, esto sumado a la presencia continua de vectores artrópodos en dichos galpones antes y después de iniciada la crianza aumenta la transmisión horizontal. Todo esto y el poco tiempo de descanso que se da en algunos galpones debido a la demanda de pollos por parte del mercado consumidor, hacen que sea posible un número mayor de ooquistes en cama, sin embargo esta contaminación previa a la crianza no representa riesgo para el lote; ya que se debe tomar en cuenta que las camas como parte de preparación del inicio de una nueva campaña son sometidas al flameado intenso, por lo que los ooquistes encontrados no necesariamente son viables, es decir, infectivos a las aves, como fue observado en el plantel A.
Con relación a los resultados encontrados en los planteles que tuvieron el pico de contaminación de ooquistes a los 28 días, se observó que los animales fueron menos pigmentados en comparación con los que tuvieron su pico de contaminación a los 42 días. Esto puede ser atribuido a que los animales no asimilaron la cantidad necesaria de pigmentantes en la etapa donde la absorción intestinal está en su máximo nivel de eficiencia, considerando que el ave aprovecha más los nutrientes con el objetivo de obtener mayor producción cárnica y buena pigmentación.

Las observaciones de campo indican mejor pigmentación en machos que en hembras. En nuestro estudio se encontró mayor intensidad de color en machos de algunos galpones que en hembras (Cuadro 2) sin embargo, esta diferencia no fue estadísticamente significativa. Las razones por las que puede haber mayor pigmentación en machos que en hembras se debería al mayor consumo de alimento en los machos, en consecuencia a un mayor aporte de pigmentos al ave, lo que dará un color más intenso que en las hembras. Otro factor mencionado por Piraces y Cortez (1994), es èl engrasamiento del ave, el cual favorece el depósito de xantófilas, que da un mejor color al animal, habiendo diferencias entre la capacidad de engrase entre hembras y machos. 
La presencia de coccidiosis subclínica en todas las granjas en estudio, no produjo mayores alteraciones en la pigmentación y en los parámetros productivos, debido posiblemente a lo expresado por Bedrnik (1999), que los animales que excretan un alto número de ooquistes, pueden estar clínicamente sanos siempre y cuando no existan condiciones desfavorables que afecten a las aves, gracias al buen manejo.

En el trabajo se encontró una baja correlación $(r=-0.098)$ entre la intensidad de pigmentación del ave y el número de ooquistes por gramo de cama. Esto puede deberse a que el recuento de ooquistes en cama sirve como un medio de estudio que mide contaminación por ooquistes en cama, para obtener una impresión general de la granja respecto a la coccidiosis, pero no identifica especies de Eimeria ni viabilidad de la misma. Estos 2 últimos puntos son muy importantes ya que la disminución de la absorción intestinal está relacionada con ciertas especies de Eimerias, primordialmente E. acervulina y E. máxima, que afectan el intestino anterior, provocando con ello una disminución en la absorción de carotenoides y produciendo a su vez aves con baja pigmentación. Por otro lado, el flameado afecta la viabilidad e inhibe la esporulación del ooquiste.

Villegas en un estudio realizado en 1983, encontró que el número de ooquistes en el contenido fecal no siempre debe ser interpretado como un factor para evaluar la severidad de la coccidiosis en la parvada. Exposiciones relativamente ligeras de ooquistes de E. máxima podrían afectar mucho más el rendimiento del pollo que muchos miles de ooquistes de E. acervulina (Piraces y Cortez, 1994).

Experiencias de campo realizadas por Koning (1994), concluyeron que las infecciones subclínicas no poseen una influencia negativa sobre la productividad del ave; así tambien Reyna et.al (1983), encontraron que cantidades aproximadas de 25,000 ooquistes por gramo de cama, no afectan los parámetros productivos, si es que estos factores son correctamente manipulados para evitar la manifestación de la coccidiosis aviar. Por el contrario, Ruff (1991) menciona que niveles bajos de coccidiosis pueden tener efectos adversos significativos sobre los parámetros, por lo cual se podría decir que un adecuado manejo de los factores involucrados en la presentación de la enfermedad puede evitar la presencia de la misma.

Se debe mencionar que la medición de la intensidad de pigmentación es realizada de acuerdo a la apreciación visual del individuo. Esta medición tiene la facilidad de ser rápida y sencilla pero al mismo tiempo conlleva a un factor de error, por lo que es necesario capacitar al personal en la utilización del abanico, colocándolo para ello sobre la misma área de observación y bajo similar intensidad de luz, evitando de esta manera los errores en la toma de datos de pigmentación en pollos de carne.

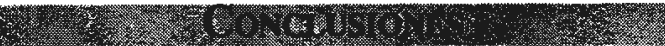

- Se encontró una baja correlación entre la pigmentación de la piel de las aves en estudio y la cantidad de ooquistes encontrados en la cama del galpón.

- En todos los galpones de los planteles evaluados se encontró presencia de coccidiosis subclínica, sin embargo, posiblemente el correcto manejo de las camas, microclima, bioseguridad, programa anticoccidial y estado de salud de las aves estudiadas, no permitieron la presentación de manifestaciones clínicas que afecten los parámetros productivos ni la pigmentación de la piel de las aves.

- El recuento de ooquistes permite tener un panorama general sobre el estado del plantel con respecto a la coccidiosis, pero no brinda un correcto diagnóstico sobre la severidad de la enfermedad. 


\section{(2)}

\section{Lamoura Chub}

1. Bedrnik, P. 1999. La coccidiosis y la inmunosupresión en las aves. Resumen del taller de Inmunosupresión. LimaPerú. p. 4-5.

2. Daniel, W. 1996. Bioestadística, base para el análisis de las ciencias de la salud. 3ra. Ed., p. 54-90. Editorial Limusa S.A. México.

3. Koning, V. 1994. ¿Eimeria, quo vadimus? World poultry. Edición especial sobre coccidiosis, Paises Bajos. p.4-6.

4. Piraces, F. y R. Cortez. 1994. Factores que afectan la pigmentación del pollo de carne. Memorias del seminario de avicultura , México.
5. Reyna, P., et al. 1983. Survival of coccidia in poultry litter and reservoirs of infection. Avian Diseases. U.S.A: 27: 464473.

6. Rojas, M. 1990. Parasitismo de rumiantes domésticos. Terapia, prevención y modelos para el aprendizaje. 1a. ed., p.367-370. Ed. Maijosa, Perú.

7. Ruff, M. 1991. Interacción de bajos niveles de coccidiosis con otras enfermedades. Avicultura Profesional. México. 9(1):15-20.

8. Villegas, P. 1983. Coccidiosis. Memorias del V Seminario Internacional de Patología Aviar. U.S.A. p. 103-121. 